\title{
New Solution of Substrate Concentration in the Biosensor Response by Discrete Homotopy Analysis Method
}

\author{
Seyyed Ali Madani Tonekaboni ${ }^{1}$, Ali Shahbazi Mastan Abad $^{2}$, Shahab Karimi ${ }^{2}$, Mitra Shabani ${ }^{2}$ \\ ${ }^{1}$ School of Mechanic, University of Waterloo, Ontario, Canada; ${ }^{2}$ School of Mechanics, University of Tehran, Tehran, Iran. \\ Email: ali.madani.1368@gmail.com, ali_shahbazi1990@yahoo.com, akharinpesar69@yahoo.com,mitrashabani@ymail.com \\ Received June 22 ${ }^{\text {nd }}, 2013$; revised July $28^{\text {th }}, 2013$; accepted August 26 ${ }^{\text {th }}, 2013$ \\ Copyright (C) 2013 Seyyed Ali Madani Tonekaboni et al. This is an open access article distributed under the Creative Commons At- \\ tribution License, which permits unrestricted use, distribution, and reproduction in any medium, provided the original work is prop- \\ erly cited.
}

\begin{abstract}
In this article, Discrete Homotopy Analysis Method (DHAM), as a new numerical method, is employed to investigate amperometric biosensor at mixed enzyme kinetics and diffusion limitation. Mathematical modeling of the problem is developed utilizing non-Michaelis-Menten kinetics of the enzymatic reaction. Different results are obtained for different values of the dimensionless parameters described in the paper. The presented solution is then compared with the available actual and simulated results.
\end{abstract}

Keywords: Discrete Homotopy Analysis Method; Amperometric Biosensor; Mathematical Modeling; Non-Michaelis-Menten Kinetics

\section{Introduction}

Biosensor is a device which measures biologically relevant information such as oxygen electrodes, neutral interfaces, etc. [1]. It is also utilized as a component of the transduction mechanisms [1]. Furthermore, it has been applied as a transducer, mapping the change in biomolecules into electrical signals [2]. Biosensors produce a signal indicative of the concentration of the measured analyte. As such, they are used in many industrial, environmental, food safety [3], and medical applications. Examples of such use are detection of pathogens [4], toxic metabolites such as mycotoxins [5], and pesticides and water contaminants such as heavy metal ions [6]. These applications showcase the wide usage and studies of biosensors and highlight the requirement of low detection limits and quicker analysis with high specificity for biosensors [2]. Mathematical modeling is widely used as an important tool to investigate and optimize the analytical characteristics of biosensors [9]. Investigative monolayer membrane contained in the model biosensors are used to study the biochemical treatment of biosensors $[7,8]$. The mathematical model developed is based on reaction-diffusion equations including none-linear terms that relate to non-Michaelis-Mentenkinetics of the enzymatic reaction $[9,10]$.

In addition to several numerical methods employed for solving linear and nonlinear differential equations, there exists some analytical methods such as perturbation method [11], $\delta$-expansion method [12], Adomian decomposition method (ADM) [13,14], and Homotopy perturbation method (HPM) [15,16]. All of the above mentioned methods including the numerical methods have certain restrictions, such as necessity for existence of small parameters, incapability of determining convergence regions, etc. One of the analytical methods proposed in the last couple of decades is homotopy analysis method (HAM) in which many of these restrictions have been omitted. In 1992, Liao introduced homotopy analysis method (HAM) for solving strongly nonlinear differential equations [17]. Using the linear property of homotopy, one can transform a nonlinear problem into an infinite number of linear sub-problems regardless of the existence of small parameterss in the original non-linear problem. HAM is a powerful mathematical technique and has already been applied to several nonlinear problems [16-22].

Since HAM has many advantages in comparison to other analytical methods, it is employed to solve continuous problems. Hence, after the discrete ADM method [23], discrete Homotopy analysis method (DHAM) was introduced in 2010 by Zhu et al. [24]. This method can be applied to complex problems containing discontinuity 
in fluid characteristics and the geometry of the problem. In addition, it needs little computational cost as a numerical method in comparison to HAM as an analytical approach. DHAM has similar advantages to continuous HAM. For instance, by means of introducing an auxiliary parameter one can adjust and control the convergence region of the solution series. This method should be employed for solving various differential equations to highlight its high capabilities in comparison with other numerical methods.

The main focus of this paper is on amperometric biosensor at mixed enzyme kinetics and diffusion limitation by utilizing DHAM as a powerfull method. Non-Michaelis-Menten kinetics of the enzymatic reaction is used to obtain the constitutive equation of the problem. Several non-dimensional parameters are defined to the dimensionless equation. The obtained non-dimensional equation is used to procure the $m^{\text {th }}$-order deformation equation as an important step towards obtaining the solution. The $h$-curves obtained for several cases are illustrated in this paper to clarify the convergence region of the solution. Finally, the obtained solution is analyzed to investigate the effects of varying each dimensionless parameter in the procured equation of the problem. In addition, some of the results are compared with the actual and simulated results available in the literature [25].

\section{Mathematical Modeling}

Spatial dependency of enzyme kinetics on biochemical systems has recently attracted much attention by considering the effect of diffusion in these processes $[9,10]$. The simplest scheme of non-Michaelis-Menten kinetics may for instance be described by adding to the Michaelis-Menten scheme (2.1) the relationship of the interaction of the enzyme substrate complex $(E S)$ with another substrate molecule $(S)$ (2.2) followed by the generation of non-active complex $\left(E S_{2}\right)$ as

$$
\begin{aligned}
& E+S \leftrightarrow E S \rightarrow E+P \\
& E S+S \leftrightarrow E S_{2}
\end{aligned}
$$

The reaction is sometimes said to display MichaelisMenten kinetics in which the relationship between the rate of an enzyme catalyzed reaction and the substrate concentration takes the form

$$
v=\frac{V_{\max }[S]}{K_{M}+[S]}
$$

where $v$ and $V_{\max }$ are the so-called "initial reaction velocity" and maximum velocity respectively.

In addition, $K_{M}$ is known as Michaelis constant for $S . K_{M}$ and $V_{\max }$ are constants at a given temperature and a given enzyme concentration.

The reactions exhibit non-Michaelis-Menten kinetics, in which the kinetic behavior does not obey the Equation
(2.3). The velocity function $v$ for the simple reaction process without competitive inhibition is given by Pao [26] and Baronas et al. [27], which is based on the nonMichaelis-Menten hypothesis,

$$
v=\frac{k_{c}[E]_{0}[S]}{K_{M}+[S]+[S]^{2} / K_{i}}=\frac{V_{\max }[S]}{K_{M}+[S]+[S]^{2} / K_{i}}
$$

where the constants $V_{\max }=k_{c}[E]_{0}, K_{M}$ and $K_{i}$ are Michaelis-Menten and inhibition constants respectively. The Equation (2.4) conforms to Equation (2.3) for large values of $K_{i}$ with respect to $K_{M}$. On the basis of Equation (2.4), the rate is maximized by increasing the concentration. It is then said to be inhibited by the substrate. In addition, the constant $K_{i}$ (which has the dimension of a concentration) is called the substrate inhibition constant. For obtaining the rate of change of substrate concentration $S=S(\eta, t)$ at time $t$ and position $\eta \in \Omega$ throughout the domain, the following equation given by Pao [26] is used.

$$
\frac{\partial S}{\partial t}=D_{S} \nabla \cdot(\nabla S)-v(\eta, t)
$$

$D_{S}$ is the substrate diffusion coefficient and $\nabla S$ is the gradient operation. On the basis of non-MichaelisMenten kinetics, Equation (2.5) becomes

$$
\frac{\partial S}{\partial t}=D_{S} \frac{\partial^{2} S}{\partial \eta^{2}}-\frac{K S}{1+S / K_{M}+S^{2} / K_{i} K_{M}}
$$

in which $K=K_{c} E_{0} / K_{M}$.

In this paper, the steady state condition is accounted for and hence, Equation (2.6) is changed to the non-dimensional form [25] using the following non-dimensional parameters

$$
\begin{gathered}
D_{S} \frac{\partial^{2} S}{\partial \eta^{2}}-\frac{K S}{1+S / K_{M}+S^{2} / K_{i} K_{M}} \\
u=\frac{S}{k s^{\infty}}, \quad \mathrm{x}=\frac{\eta}{L}, \quad \mathrm{~K}=\frac{k L^{2}}{D_{S}}=\phi^{2}, \quad \alpha=\frac{k s^{\infty}}{K_{M}}, \quad \beta=\frac{k s^{\infty}}{K_{i} K_{M}}
\end{gathered}
$$

This results in the following non-dimensional differential equation

$$
\frac{\partial^{2} u}{\partial x^{2}}-\frac{K u}{1+\alpha u+\beta u^{2}}=0, \quad 0<u \leq 1
$$

Equation (2.8) must be solved such that it satisfies the following boundary conditions

$$
\begin{array}{lll}
u=1 & \text { at } & x=1 \\
\frac{\partial u}{\partial x}=0 & \text { at } & x=0
\end{array}
$$

\section{Analytical and Numerical Solutions DHAM Solution}

The discrete form of the nonlinear differential equation 
(Equation (2.8)) is obtained as the first step of DHAM's procedure of the solution

$$
\begin{aligned}
N\left[\varphi_{i}(q)\right]= & \left(1+\alpha \varphi_{i}(q)+\beta\left(\varphi_{i}(q)\right)^{2}\right) \\
& \frac{\varphi_{i-1}(q)-2 \varphi_{i}(q)+\varphi_{i+1}(q)}{\Delta x^{2}}-K \varphi_{i}(q)
\end{aligned}
$$

where $i$ is the node number, $N$ is the nonlinear operator, and the function $\varphi_{i}(q)$ is defined as

$$
\begin{aligned}
& \lim _{q \rightarrow 0} \varphi_{i}(q)=u_{0, i}(X), \\
& \lim _{q \rightarrow 1} \varphi_{i}(q)=u_{i}
\end{aligned}
$$

where $u_{i}$ is the unknown field variable at node number $i, q \in(0,1)$ is the embedding parameter, and $u_{0, i}$ is the initial guess which is employed to meet the requirements of the boundary conditions. Here, $U_{0, i}$ is valued at " 1 " satisfying all the boundary conditions stated in Equation (2.9).

Through the generalizing concept of DHAM, the socalled zero-order deformation equation can be written as

$$
(1-q) L\left[\varphi_{i}(q)-u_{0, i}\right]=q h H_{i} N\left[\varphi_{i}(q)\right]
$$

where $h \neq 0$ is the non-zero auxiliary parameter, $H_{i}$ is the auxiliary function, and $L$ is the auxiliary linear operator which is chosen here as

$$
\mathcal{L}\left(f_{i}\right)=\frac{f_{i-1}-2 f_{i}+f_{i+1}}{\Delta x^{2}}
$$

Expanding $\varphi_{i}(q)$ in Taylor series with respect to the embedding parameter $q$, one obtains

$$
\begin{aligned}
& \varphi_{i}(q)=u_{0, i}+\sum_{m=1}^{\infty} u_{m, i} q^{m} \\
& u_{m, i}=\left.\frac{1}{m !} \frac{\partial^{m} \varphi_{i}(q)}{\partial q^{m}}\right|_{q=0}
\end{aligned}
$$

With due attention to the procedure of DHAM [27], $u_{m, i}$ should be chosen so as the following equation is satisfied

$$
\frac{3 u_{m, 0}-4 u_{m, i}+u_{m, i}}{2 \Delta x}=u_{m}(1)=0
$$

If the series $\varphi_{i}(q)$ converges at $q=1$, then the series solution is

$$
\varphi_{i}(1)=u_{0, i}+\sum_{m=1}^{\infty} u_{m, i}
$$

where $u_{m, i}$ could be obtained by the so-called highorder deformation equation. For obtaining the $m^{\text {th }}$-order deformation equation, the following vector is defined as

$$
\boldsymbol{u}_{n}=\left\{u_{0, i}, u_{1, i}, \cdots, u_{n, i}\right\}
$$

Differentiating both sides of the zero-order equation $m$ times with respect to $q$ and then setting $q=0$, the socalled $m^{\text {th }}$-order deformation equation can be obtained as

$$
L\left[u_{m, i}-\chi_{m} u_{m-1, i}\right]=h H_{i} R_{m, i}\left(\boldsymbol{u}_{m-1}\right)
$$

where

$$
\begin{aligned}
& \chi_{m}=\left\{\begin{array}{l}
0, \\
1, \quad \text { otherwise }
\end{array}\right. \\
& R_{m, i}\left(\boldsymbol{u}_{m-1}\right)=\left.\frac{1}{(m-1) !} \frac{\partial^{m-1} N\left[\varphi_{i}(q)\right]}{\partial q^{m-1}}\right|_{q=0}
\end{aligned}
$$

Therefore, the following relation is obtained

$$
\begin{aligned}
R_{m, i}\left(\boldsymbol{u}_{m-1}\right)= & u_{m-1}^{\prime \prime}+\alpha \sum_{j=0}^{m-1} u_{j} u_{m-1-j}^{\prime \prime} \\
& +\beta \sum_{j=0}^{m-1} u_{m-1-j} \sum_{k=0}^{j} u_{k} u_{j-k}^{\prime \prime}-K u_{m-1}
\end{aligned}
$$

We are free to choose the auxiliary parameter $h$, the auxiliary function $H_{i}$, the initial guess $u_{0, i}$, and the auxiliary linear operator $L$ so that the validity and flexibility of the DHAM solution to control the convergence region is proven. Due to the rule of solution expression [27], the auxiliary function is chosen as follows

$$
H_{i}=1
$$

According to the DHAM, the valid region of the auxiliary parameter $h$ for convergence of the solution series is the flat regions of $h$-curves. To see the proper values of $h$, the $h$-curves are plotted for different values of dimensionless parameters $\alpha, \beta$ and $K$ in Figure 1 for obtaining the valid results for the considered conditions.

\section{Results and Discussion}

The procedure for solving the non-dimensional equation of enzyme reaction (Equation (2.8)) which is based on the non-Michaelis-Menten kinetics theory utilizing DHAM is described in the Section 3. It is mentioned there that the $m^{\text {th }}$-order deformation equation should be employed to solve the problem. As the first step towards the solution, the diagrams for variation of non-dimensional parameter $u(X)$ versus auxiliary parameter $h$ for different investigated cases are illustrated in Figure 1. Then, the flat region of $h$-curves in each case is obtained from these diagrams.

On the basis of the chosen values of auxiliary parameter $h$ in the flat regions of $h$-curves (Figure 1), some useful diagrams including variations of $u(X)$ versus $X$ (Figure 2) are procured to clarify the dependency of these variations on different non-dimensional parameters defined in Equation (2.8). It is shown in Figure 2 that the effect of variation of non-dimensional parameter $K$ on the profiles of $u(X)$ is substantial. Values of $u(X)$ at 


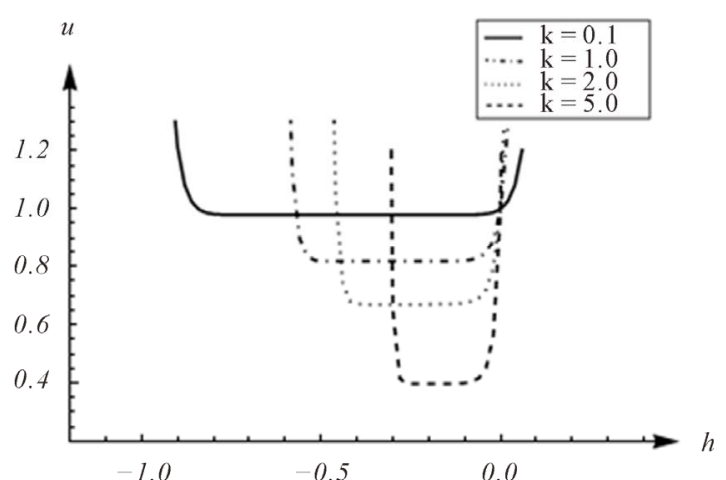

(a)

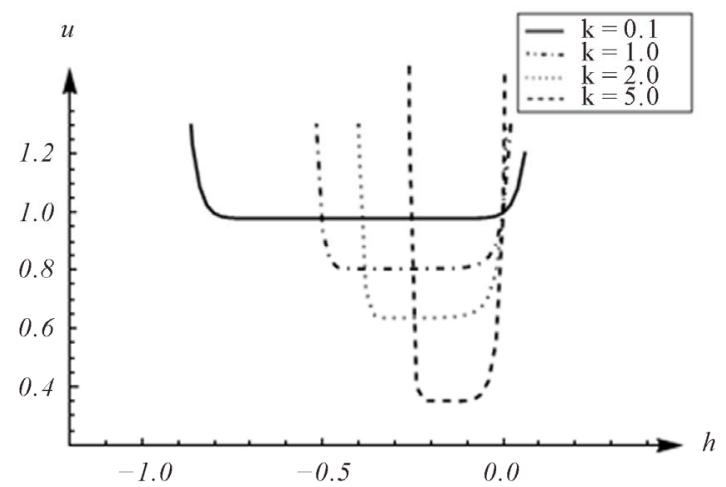

(b)

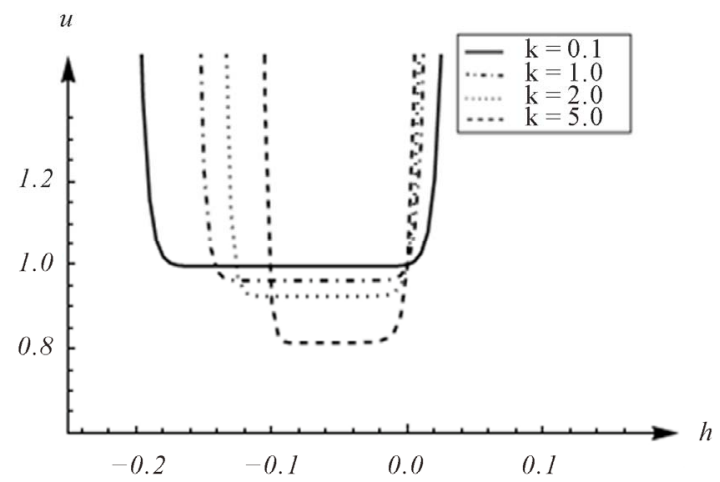

(c)

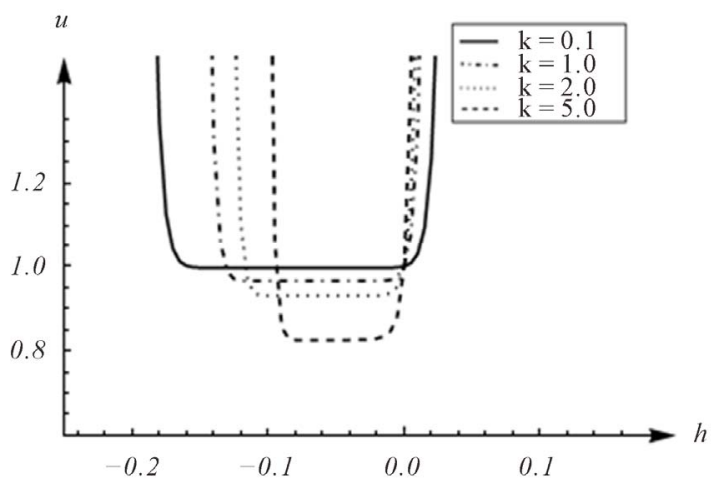

(d)

Figure 1. Variations of $u(X)$ versus non-dimensional parameter $X$ for (a) $\alpha=1.0, \beta=0.1$, (b) $\alpha=0.1, \beta=1.0$, (c) $\alpha=$ $10.0, \beta=0.1$ and (d) $\alpha=10.0, \beta=1.0$.

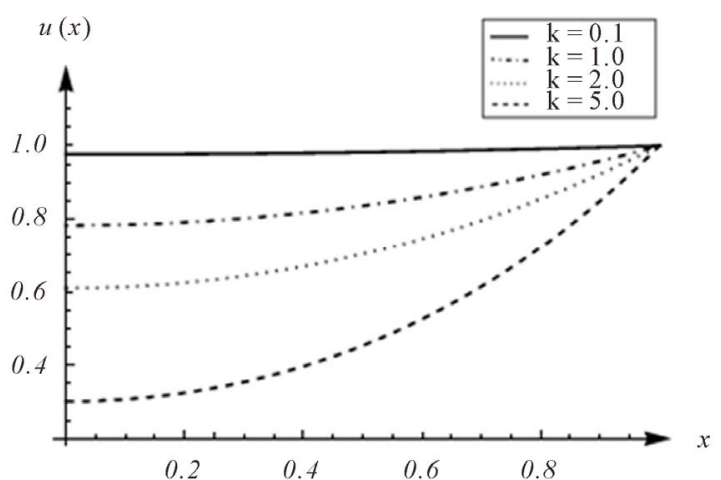

(a)

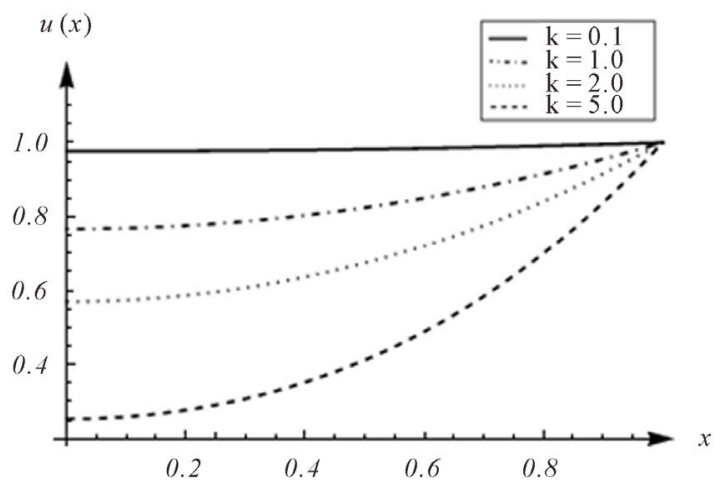

(b)

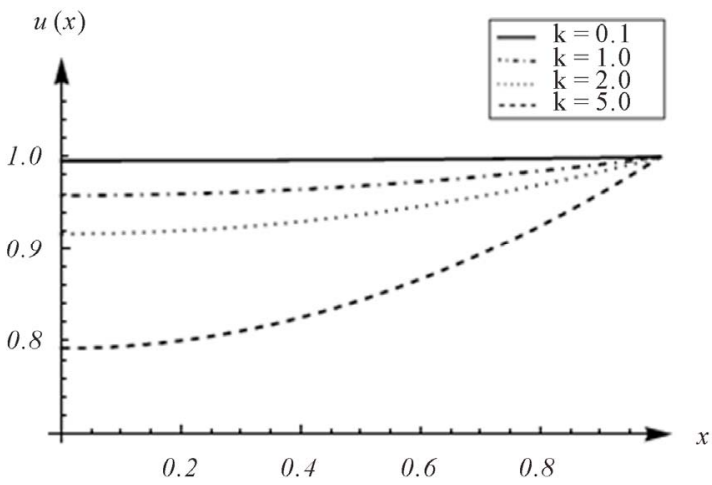

(c)

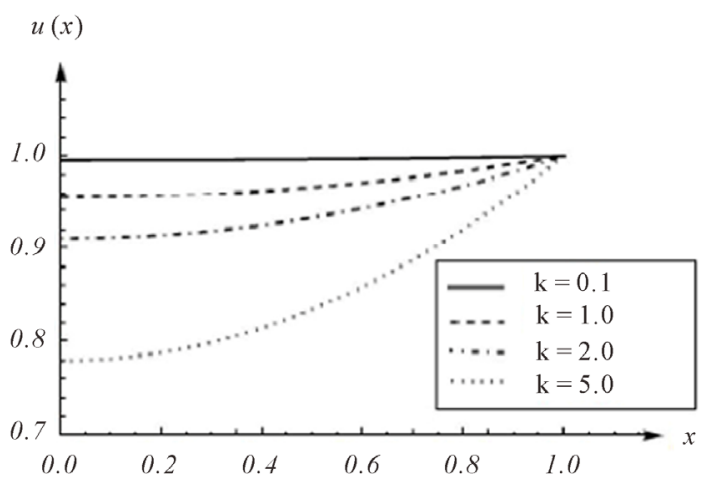

(d)

Figure 2. Variations of $u(X)$ versus auxiliary parameter $h$ for (a) $\alpha=1.0, \beta=0.1$, (b) $\alpha=0.1, \beta=1.0$, (c) $\alpha=10.0, \beta=$ 0.1 and (d) $\alpha=10.0, \beta=1.0$. 
different locations are presented in Tables 1 and 2 for better clarifying the effects of $K$, as well as other nondimensional parameter $(\alpha, \beta)$. It is clearly shown that the values of variable for lower value of $\alpha$ are lower than he higher ones. In addition, the spatial variation of variables which is also shown in Figure $\mathbf{2}$ is clarified.

\section{Verification of the Solution}

The results of the problem obtained by employing DHAM and the results procured by simulation and actual results [25] are compared in Table 3 to show the accu- racy of the presented solution. As such, the presented result in this paper can be utilized as promising data for investigating the behavior of the enzyme reaction in the considered conditions.

\section{Conclusions}

Solution to the amperometric biosensor at mixed enzyme kinetics and diffusion limitation is presented util- izing DHAM as a new numerical method. Dimensionless equation of the problem is obtained using the mathematical modeling presented in this paper, which is based on non- Michaelis-Menten kinetics of the enzymatic reaction. Solution procedure of the non-dimensional equation of enzyme reaction is described and $m^{\text {th }}$-order $d e$ formation equation is obtained on the basis of the non-dimensional enzyme reaction equation presented in this paper. Several $h$-curves are dipicted to show the convergence region of the solution. Results of the solution are presented for different quantities of the dimen sionless parameters used to non-dimensionalize the enzyme reaction equation. It is shown that the most effecttive parameter in the reaction and local dependency of the dependent variable of the problem $u(X)$ is $K$. Available results in the literature are used conclusively to prove the high accuracy of the presented solution.

On the basis of the presented solution for the considered problem in the area of enzyme kinetics, it can be concluded that DHAM can be employed to solve differ-

Table 1. Values of non-dimensional variable $u(X)$ at different locations for $\alpha=1.0, \beta=0.1$ and $\alpha=0.1, \beta=1.0$ for different values of non-dimensional parameter $K$.

\begin{tabular}{ccccccccc}
\hline$x$ & \multicolumn{9}{c}{$\alpha=1.0, \beta=0.1$} & \multicolumn{4}{c}{$\alpha=0.1, \beta=1.0$} \\
\cline { 2 - 9 } & $K=0.1$ & $K=1.0$ & $K=2.0$ & $K=5.0$ & $K=0.1$ & $K=1.0$ & $K=2.0$ & $K=5.0$ \\
\hline 0 & 0.9764 & 0.7831 & 0.6097 & 0.3012 & 0.9762 & 0.7675 & 0.5694 & 0.2532 \\
0.2 & 0.9773 & 0.7916 & 0.6246 & 0.3244 & 0.9771 & 0.7767 & 0.5860 & 0.2770 \\
0.4 & 0.9802 & 0.8172 & 0.6695 & 0.3967 & 0.9800 & 0.8044 & 0.6360 & 0.3521 \\
0.6 & 0.9849 & 0.8601 & 0.7457 & 0.5255 & 0.9848 & 0.8507 & 0.7207 & 0.4884 \\
0.8 & 0.9915 & 0.9209 & 0.8551 & 0.7221 & 0.9914 & 0.9159 & 0.8416 & 0.7000 \\
1.0 & 1.0000 & 1.0000 & 1.0000 & 1.0000 & 1.0000 & 1.0000 & 1.0000 & 1.0000 \\
\hline
\end{tabular}

Table 2. Values of non-dimensional variable $u(X)$ at different locations for $\alpha=10.0, \beta=0.1$ and $\alpha=10.0, \beta=1.0$ for different values of non-dimensional parameter $K$.

\begin{tabular}{ccccccccc}
\hline \multirow{2}{*}{$x$} & \multicolumn{9}{c}{$\alpha=10.0, \beta=0.1$} & \multicolumn{4}{c}{$\alpha=10.0, \beta=1.0$} \\
\cline { 2 - 9 } & $K=0.1$ & $K=1.0$ & $K=2.0$ & $K=5.0$ & $K=0.1$ & $K=1.0$ & $K=2.0$ & $K=5.0$ \\
\hline 0 & 0.9955 & 0.9551 & 0.9105 & 0.7790 & 0.9958 & 0.9583 & 0.9167 & 0.7924 \\
0.2 & 0.9957 & 0.9569 & 0.9141 & 0.7878 & 0.9960 & 0.9600 & 0.9200 & 0.8007 \\
0.4 & 0.9962 & 0.9623 & 0.9248 & 0.8142 & 0.9965 & 0.9650 & 0.9300 & 0.8255 \\
0.6 & 0.9971 & 0.9712 & 0.9427 & 0.8583 & 0.9973 & 0.9733 & 0.9467 & 0.8670 \\
0.8 & 0.9984 & 0.9838 & 0.9678 & 0.9202 & 0.9985 & 0.9850 & 0.9700 & 0.9252 \\
1.0 & 1.0000 & 1.0000 & 1.0000 & 1.0000 & 1.0000 & 1.0000 & 1.0000 & 1.0000 \\
\hline
\end{tabular}

Table 3. Comparison of results of the DHAM with simulation and actual results of the problem at different location and for different values of non-dimensional parameter $K$.

\begin{tabular}{|c|c|c|c|c|c|c|c|c|c|}
\hline \multirow{2}{*}{$x$} & \multicolumn{3}{|c|}{$K=0.1$} & \multicolumn{3}{|c|}{$K=0.1$} & \multicolumn{3}{|c|}{$K=5.0$} \\
\hline & Simulation & DHAM & Actual & Simulation & DHAM & Actual & Simulation & DHAM & Actual \\
\hline 0 & 0.9500 & 0.9520 & 0.9520 & 0.6500 & 0.6481 & 0.6481 & 0.2100 & 0.2113 & 0.2113 \\
\hline 0.25 & 0.9529 & 0.9550 & 0.9550 & 0.6666 & 0.6684 & 0.6684 & 0.2502 & 0.2452 & 0.2452 \\
\hline 0.50 & 0.9618 & 0.9639 & 0.9639 & 0.7295 & 0.7308 & 0.7308 & 0.3585 & 0.3578 & 0.3578 \\
\hline 0.75 & 0.9767 & 0.9789 & 0.9789 & 0.8386 & 0.8390 & 0.8390 & 0.5893 & 0.5851 & 0.5851 \\
\hline 1.0 & 0.9976 & 1.0000 & 1.0000 & 0.9940 & 1.0000 & 1.0000 & 0.9970 & 1.0000 & 1.0000 \\
\hline
\end{tabular}


ent nonlinear ordinary differential equations used to model different problems in Engineering and Science. The accuracy is clearly shown and the ablility of the aproach to control the convergence of the solution is obviously shown. Therefore, the employed method not only can be used to solve different complicated nonlinear problems but also can be considered as a promising numerical technique.

\section{REFERENCES}

[1] F. Scheller and F. Schubert, "Biosensors," Vol. 7, Elsevier, Amsterdam, 1988.

[2] U. Wollenberger, F. Lisdat and F. W. Scheller, "Enzymatic Substrate Recycling Electrodes. Frontiers in Biosensorics. B and II, Practical Applications,” Birkhauser Verlag, Basel, 1997, pp. 45-70.

[3] A. Baeumner, Analytical and Bioanalytical Chemistry, Vol. 377, 2003, pp. 434-445.

[4] M. Pohanka, P. Skladal and M. Kroca, "Biosensors for Biological Warfare Agent Detection,” Defense Science Journal, Vol. 57, No. 3, 2007, pp. 185-93.

[5] M. Pohanka, D. Jun and K. Kuca, "Mycotoxin Assay Using Biosensor Technology: A Review,” Drug and Chemical Toxicology, Vol. 30, No. 3, 2007, pp. 253-261. http://dx.doi.org/10.1080/01480540701375232

[6] S. Haron and A. K. Ray, "Optical Biodetection of Cadmium and Lead Ions in Water," Medical Engineering and Physics, Vol. 28, 2006, No. 10, pp. 978-981.

[7] A. J. Baeumner, C. Jones, C.Y. Wong and A. Price, “A Generic Sandwich-Type Biosensor with Nanomolar Detection Limits," Analytical and Bioanalytical Chemistry, Vol. 378, No. 6, 2004, pp. 1587-1593. http://dx.doi.org/10.1007/s00216-003-2466-0

[8] T. Schulmeister, "Mathematical Modeling of the Dynamic Behavior of Ampero-Metric Enzyme Electrodes," Selective Electrode Reviews, Vol. 12, 1990, pp. 203-260.

[9] R. Aris, "The Mathematical Theory of Diffusion and Reaction in Permeable Cat-alysts. The Theory of the Steady State,” Clarendon Press, Oxford, 1975.

[10] L. K. Bieniasz and D. Britz, "Recent Developments in Digital Simulation of Electroan-Alytical Experiments," Polish Journal of Chemistry, Vol. 78, 2004, pp. 11951219.

[11] J. D. Cole, "Perturbation Methods in Applied Mathematics,” Blaisdel, Waltham, 1968.

[12] A. V. Karmishin, A. I. Zhukov and V. G. Kolosov, "Methods of Dynamics Calculation and Testing for ThinWalled Structures,” Mashinostroyenie, Moscow, 1990.

[13] G. Adomian, "Solving Frontier Problems of Physics: The Decomposition Method,” Kluwer Academic Publishers, Boston, 1994. http://dx.doi.org/10.1007/978-94-015-8289-6

[14] G. Adomian, "A Review of the Decomposition Method in Applied Mathematics,” Journal of Mathematical Analysis and Applications, Vol. 135, No. 2, 1998, pp. 501-544. http://dx.doi.org/10.1016/0022-247X(88)90170-9
[15] J. H. He, "Homotopy Perturbation Technique," Computer Methods in Applied Mechanics and Engineering, Vol. 178, No. 3-4, 1999, pp. 257-262. http://dx.doi.org/10.1016/S0045-7825(99)00018-3

[16] J. H. He, “An Approximate Solution Technique depEnding upon an Artificial Parameter," Communications in Nonlinear Science and Numerical Simulation, Vol. 3, No. 2, 1998, pp. 92-97. http://dx.doi.org/10.1016/S1007-5704(98)90070-3

[17] S. J. Liao, “The Proposed Homotopy Analysis Technique for the Solution of Nonlinear Problems,” PhD Thesis, Shanghai Jiao Tong University, 1992.

[18] S. Abbasbandy, "The Application of Homotopy Analysis Method to Nonlinear Equations Arising in Heat Transfer," Physics Letters A, Vol. 360, No. 1, 2006, pp. 109113. http://dx.doi.org/10.1016/j.physleta.2006.07.065

[19] S. J. Liao, "Beyond Perturbation: Introduction to Homotopy Analysis Method,” Chapman \& Hall/CRC Press, Boca Raton, 2003. http://dx.doi.org/10.1201/9780203491164

[20] J.-H. He, “Comparison of Homotopy Perturbation Method and Homotopy Analysis Method," Applied Mathematics and Computation, Vol. 156, No. 2, 2004, pp. $527-$ 539. http://dx.doi.org/10.1016/j.amc.2003.08.008

[21] S. J. Liao, "A Short Review on the Homotopy Analysis Method in Fluid Mechanics," Journal of Hydrodynamics, Ser. B, Vol. 22, No. 5, 2010, pp. 882-884.

[22] S. J. Liao,” On the Homotopy Analysis Method for Nonlinear Problems", Applied Mathematics and Computation, Vol. 147, No. 2, 2004, pp. 499-513. http://dx.doi.org/10.1016/S0096-3003(02)00790-7

[23] A. Bratsos, M. Ehrhardt and I. T. Famelis, "A Discrete Adomian Decomposition Method for Discrete Nonlinear Schrödinger Equations," Applied Mathematics and Computation, Vol. 197, No. 1, 2008, pp. 190-205. http://dx.doi.org/10.1016/j.amc.2007.07.055

[24] H. Q. Zhu, H. Z. Shu and M. Y. Ding, "Numerical Solutions of Partial Differential Equations by Discrete Homotopy Analysis Method,” Applied Mathematics and Computation, Vol. 216, No. 12, 2010, pp. 3592-3605. http://dx.doi.org/10.1016/j.amc.2010.05.005

[25] M. Daniel, "Dunlavy Ph.D. Candidacy Prospectus, A Homotopy Method for Predicting the Lowest Energy Conformations of Proteins,” April 18, 2003.

[26] P. Manimozhia, A. Subbiahb and L. Rajendrana, "Solution of Steady-State Substrate Concentration in the Action of Biosensor Response at Mixed Enzyme Kinetics," Sensors and Actuators B, Vol. 147, No. 1, 2010, pp. 290-297. http://dx.doi.org/10.1016/j.snb.2010.03.008

[27] C. V. Pao, "Mathematical Analysis of Enzyme-Substrate Reaction Diffusion Insomebiochemical Systems," Nonlinear Analysis: Theory, Vol. 4, No. 2, 1979, pp. 369-392. http://dx.doi.org/10.1016/0362-546X(80)90061-9 